FTPI-MINN-16/36, UMN-TH-3616/16

\title{
(In)dependence of $\theta$ in the Higgs Regime without Axions
}

\author{
Mikhail Shifman ${ }^{a, b}$ and Arkady Vainshtein ${ }^{a, b, c}$ \\ ${ }^{a}$ School of Physics and Astronomy, University of Minnesota, Minneapolis, MN \\ 55455, USA \\ ${ }^{b}$ William I. Fine Theoretical Physics Institute, University of Minnesota, \\ Minneapolis, MN 55455, USA \\ ${ }^{c}$ Kavli Institute for Theoretical Physics, University of California, Santa Barbara, \\ CA 93106, USA
}

\begin{abstract}
We revisit the issue of the vacuum angle $\theta$ dependence in weakly coupled (Higgsed) Yang-Mills theories. Two most popular mechanisms for eliminating physical $\theta$ dependence are massless quarks and axions. Anselm and Johansen noted that the vacuum angle $\theta_{\mathrm{EW}}$, associated with the electroweak $\mathrm{SU}(2)$ in the Glashow-WeinbergSalam model (Standard Model, SM), is unobservable although all fermion fields obtain masses through Higgsing and there is no axion. We generalize this idea to a broad class of Higgsed Yang-Mills theories.

In the second part we consider consequences of Grand Unification. We start from a unifying group, e.g. SU(5), at a high ultraviolet scale and evolve the theory down within the Wilson procedure. If on the way to infrared the unifying group is broken down into a few factors, all factor groups inherit one and the same $\theta$ angle - that of the unifying group. We show that embedding the $\mathrm{SM}$ in $\mathrm{SU}(5)$ drastically changes the Anselm-Johansen conclusion: the electroweak vacuum angle $\theta_{\mathrm{EW}}$, equal to $\theta_{\mathrm{QCD}}$ becomes in principle observable in $\Delta B=\Delta L= \pm 1$ processes. We also note in passing that if the axion mechanism is set up above the unification scale, we have one and the same axion in the electroweak theory and $\mathrm{QCD}$, and their impacts are interdependent.
\end{abstract}




\section{Introduction}

Shortly after introduction of the vacuum angle $\theta$ in QCD and in more generic YangMills theories [1,2 it was realized that the origin of the vacuum angle and its implications are determined by physics at the ultraviolet (UV) scale [3]. Since a nonvanishing $\theta$ in $\mathrm{QCD}$ implies that $C P$ is not conserved in strong interactions the axion mechanism of $\theta$ screening was invented [4,5. Another way out is a spontaneous breaking of $C P$ invariance which leads to smallness of the effective low-energy vacuum angle [6, [7].

In the Standard Model $(\mathrm{SM})$ based on the $\mathrm{SU}(3) \times \mathrm{SU}(2) \times \mathrm{U}(1)$ group there is another vacuum angle $\theta_{\mathrm{EW}}$, associated with $\mathrm{SU}(2)_{\text {weak }}$. Although the related effects are exponentially suppressed the problem was studied by Anselm and Johansen in 8 (see also [9]), with the conclusion that $\theta_{\mathrm{EW}}$ is unobservable per se.

Here we would like to address the issue of vacuum angles and their observability in Higgsed theories in a more general aspect. First and foremost, we assume that at low energy scales no massless fermions are left in the theory. Second, we do not allow for the axion mechanism to be introduced. Under these conditions we raise the question: can one generalize the Anselm-Johansen mechanism making the $\theta$ angle physically unobservable? The answer is positive. We prove that in a potentially broad class of Higgsed Yang-Mills theories (with no massless fermions and no axion fields) the vacuum angle $\theta$ can be absorbed in the field redefinition and is unobservable in principle.

Then we turn to the second question: $\theta$ observability from the standpoint of grand unification theories. We assume that at high energies all gauge symmetries of the model under consideration are unified in a simple group $G$. Then, using the Wilsonean approach we descend down to low energies where some of the symmetries can be spontaneously broken, $G \rightarrow G_{1} \times G_{2} \times \ldots$, (i.e. some gauge bosons become Higgsed). The very fact of Grand Unification imposes constraints on the $\theta$-induced effects at low energies. First, above the unification scale the vacuum angle is determined by a single parameter. When we descend down in a controllable way this parameter does not split in two independent parameters [10], rather all surviving gauge groups $G_{1,2, .}$ inherit it from $G$. Thus, if a mechanism could be found to screen $\theta$ at short distances, then this mechanism would eliminate $\theta$ in all $G_{1,2, . .}$ subgroups simultaneously.

As an example we will consider SU(5) unification keeping for simplicity only one generation. We show that, in contradistinction with SM, weak $\theta$ effects are in principle observable. The Anselm-Johansen argument [8] based on the analysis of the Glashow-Weinberg-Salam model fails in SU(5). We explain why this happens. 
We also demonstrate that the effective $\theta$ are the same in the strong $\mathrm{SU}(3)$ and in the weak $\mathrm{SU}(2)$.

Then we make a brief comment on the general axion mechanism. If the latter is set up above the unification scale, we have one and the same axion in the electroweak theory and QCD, and it leads to screening vacuum angles in both sectors.

We will start with a brief review of some basic points and the original AnselmJohansen argument (see also [9, 11]). Section 2 presents generalities. In Sec.3 we construct generalizations in a broad class of Higgsed Yang-Mills theories. In Sec.4 we discuss the impact of Grand Unification. In this section we argue that the $\theta$ dependence unobservable in the Glashow-Weinberg-Salam model becomes observable if the latter is embedded in Grand Unification. In this case instead of two independent $\theta$ angles - one in QCD and another in the electroweak theory - we deal with a single vacuum angle. In Sec.4 we also make an explanatory remark about another possible source of $C P$ violation (through Kobayashi-Maskawa matrices).

\section{Generalities: $\theta$ angle and its consequence}

Let us remind that in any four-dimensional Yang-Mills theory, based on a simple group $G$ with a nontrivial homotopy $\pi_{3}(G)=Z$, an additional parameter appears: the vacuum angle $\theta_{G}$. In the Lagrangian formulation the so called $\theta$-term is

$$
\Delta \mathcal{L}_{\theta}=\frac{\theta_{G}}{32 \pi^{2}} \mathcal{F}_{\mu \nu}^{A} \widetilde{\mathcal{F}}^{\mu \nu, A},
$$

where $\mathcal{F}_{\mu \nu}^{A}$ is the gauge field strength tensor and $A$ is the index of the adjoint representation. Although this term is a total derivative it affects physics due to nonperturbative instanton effects. Note that the observable nature of the vacuum angle shows up also in the Higgs regime where the theory is at weak coupling.

Particularly, in SM the gauge group is the product $\mathrm{SU}(3) \times \mathrm{SU}(2) \times \mathrm{U}(1)$; hence, a priori we deal with two angles: $\theta_{\mathrm{QCD}}$, associated with color, and $\theta_{\mathrm{EW}}$, associated with weak $\mathrm{SU}(2)$. There are no instantons in $\mathrm{U}(1)$ so it does not give rise to another angle.

While vacuum angle parameters are certainly observable and bring in $C P$ violation in purely bosonic models (i.e. non-Abelian vectors plus Higgs fields and possibly scalars), introduction of fermion fields has a crucial impact on $\theta$-related effects. The fate of the latter drastically depends on fermionic matter content of the theory.

Generically, the fermion fields are described by a set $\psi_{\alpha}^{a}$ of the left-handed Weyl spinors plus the complex conjugated set $\bar{\psi}_{a, \dot{\alpha}}$ of right-handed spinors. The indices 
$\alpha, \dot{\alpha}=1,2$ are spinorial while the index $a$ refers to a representation of the gauge group $G$ which can be reducible. The bilinear fermionic Lagrangian takes the form

$$
\mathcal{L}_{F}=i \bar{\psi}^{\dot{\alpha}} \mathcal{D}_{\alpha \dot{\alpha}} \psi^{\alpha}-\left[\psi_{\alpha}^{a} M_{a b} \psi_{\beta}^{b} \epsilon^{\alpha \beta}+\text { H.c. }\right] .
$$

Here $\mathcal{D}_{\alpha \dot{\alpha}}=\partial_{\alpha \dot{\alpha}}-i A_{\alpha \dot{\alpha}}^{A} T^{A}$ is the covariant derivative acting on the column of $\psi^{\alpha}$ and $M_{a b}$ is a symmetric matrix which includes possible mass terms and Yukawa couplings in the form consistent with the gauge symmetry.

In the absence of the mass terms and Yukawa couplings, $M_{a b}=0$. Addition of fermions brings in a number of $\mathrm{U}(1)$ symmetries associated with the global phase rotation for each multiplet $\psi_{P}$,

$$
\psi_{P} \longrightarrow \mathrm{e}^{\alpha_{P}} \psi_{P}
$$

transforming under the the irreducible representation $P$.

We will focus on those chiral symmetries which are maintained at the classical level but are broken by quantum anomalies. In other words, we will consider classically conserved axial currents which are not anomaly-free. Anomalies break their conservation. Moreover, nonperturbative instanton-induced effects then reveal the breaking of the anomalous global symmetries. Famous examples include the resolution of $\mathrm{U}(1)$ problem in QCD and nonconservation of baryon and lepton charges in the electroweak sector [12].

Due to these chiral anomalies the phase rotations (3) lead to variations of the kinetic part $i \bar{\psi}^{\dot{\alpha}} \mathcal{D}_{\alpha \dot{\alpha}} \psi^{\alpha}$ of the fermion Lagrangian (2) which are of the same form as the $\theta$-terms (11), i.e. the rotations (3) shift $\theta_{G}$,

$$
\theta_{G} \longrightarrow \theta_{G}-\sum_{P} 2 T(P) \alpha_{P}
$$

where where $T(P)$ is the index of the representation $P$ defined as $\operatorname{Tr}\left(T^{a} T^{b}\right)=$ $T(P) \delta^{a b}$. In the case $M_{a b}=0$, it proves that physics is independent of the $\theta$ parameters. When $M_{a b} \neq 0$ there are two scenarios. If it is possible to find a combination of phase rotations which keeps the term $\psi_{\alpha}^{a} M_{a b} \psi_{\beta}^{b} \epsilon^{\alpha \beta}$ intact while shifting the value of the original $\theta$ as in (4) then we arrive at the same statement of $\theta$-independence as in the massless case. Otherwise, one can translate $\theta$ parameters into certain phases in $M_{a b}$ which will make physics dependent on effective $\bar{\theta}$,

$$
\bar{\theta}_{G}=\theta_{G}+\arg \operatorname{Det} M_{a b} .
$$

Below we will exemplify both scenarios by particular examples. 


\section{$3 \quad \theta$-independence without massless fermions and axions}

In this section we present examples of gauge theories where the parameter $\theta$ is not observable. The unobservability is due to a certain $U(1)$ symmetry in the fermion sector. Despite this fact, all fermions are massive in these theories. Correspondingly, there is no need for axion in these theories.

We start with the Anselm-Johansen construction for SU(2) case and then consider possible generalizations.

\subsection{The standard model case, $\mathrm{SU}(2)$}

In this case left-handed fermions compose some number of $\mathrm{SU}(2)$ doublets $L_{\alpha}^{k, A}$, $k=1,2, A=1,2, \ldots$, and singlets $S_{\alpha}$. Our consideration below refers to generic $\mathrm{SU}(2)$ case, but, particularly, in framework of the Standard Model the first generation contains 4 doublets of $\mathrm{SU}(2)_{\text {weak }}$, one leptonic and three quark ones,

$$
\left(\begin{array}{c}
\nu_{\alpha} \\
e_{\alpha}
\end{array}\right), \quad\left(\begin{array}{c}
u_{\alpha}^{i} \\
d_{\alpha}^{i}
\end{array}\right), \quad i=1,2,3,
$$

and 8 singlets

$$
\nu_{\alpha}^{c}, e_{\alpha}^{c}, u_{\alpha, i}^{c}, d_{\alpha, i}^{c},
$$

where $\nu_{\alpha}^{c}$ is added to give (if necessary) a Dirac mass to neutrino.

The masses come from the Yukawa couplings with the standard doublet Higgs field $\phi^{i}$,

$$
\mathcal{L}_{\mathrm{Y}}=h_{A B} \bar{\phi}_{k} L_{\alpha}^{k, A} S_{\beta}^{B} \epsilon^{\alpha \beta}+\tilde{h}_{A B} \epsilon_{i k} \phi^{i} L_{\alpha}^{k, A} S_{\beta}^{B} \epsilon^{\alpha \beta}+\text { H.c. . }
$$

The phase rotations of singlets are not anomalous and can be chosen to be opposite to the rotations of the doublets. Keeping the Higgs field $\phi$ intact, we see that the fermion mass terms are invariant while the kinetic part produces a change of $\theta$, see Eq. (4). It proves $\theta$-independence and the absence of the associated $C P$ non-invariance in the $\mathrm{SU}(2)$ theory (with no massless fermions and no axion).

Although electroweak instantons are exponentially suppressed in the electroweak $\mathrm{SU}(2)$ example it is interesting as a matter of principle. Besides it could be relevant to early cosmological time [13].

It is also instructive to demonstrate the phenomenon in terms of the instanton calculus. The 't Hooft interaction generated by antiinstanton [12] is

$$
\Delta \mathcal{L}_{\text {inst }} \propto \prod_{A} L_{k}^{k, A} e^{-i \theta} \exp \left(-\frac{8 \pi^{2}}{g^{2}}\right)
$$


where we accounted for the $\delta_{k}^{\alpha}$ structure of the fermion zero modes before averaging over the instanton $\mathrm{SU}(2)$ orientations. It is clear that one can eliminate the phase factor $\exp (-i \theta)$ by redefining the $L$ fields. The same conclusion follows from the fact that only instantons contribute to processes which breaks the baryon and lepton quantum numbers, $\Delta B=\Delta L= \pm 1$, in the Standard Model. Then $\exp (-i \theta)$ enters as an overall factor in the amplitude and no interference term appears for $\theta$-dependence to show up.

Thus, we see that in SM the vacuum angle $\theta_{\mathrm{EW}}$ is unobservable. This is related to the anomalous $\mathrm{U}(1)$ symmetry which is $B+L$ in this case, while $B-L$ is not anomalous.

\subsection{Generalization to $\mathrm{SU}(N)$}

Here we present a simple $\mathrm{SU}(N)$ generalization $(N>2)$ of the Anselm-Johansen construction. Consider $\mathrm{SU}(N)$ gauge theory with $N$ complex Higgs fields $\phi_{a}^{i}$, each in the fundamental representation of $\mathrm{SU}(N)_{\text {gauge }}$. Here $i=1,2, \ldots, N$ is the color index while $a=1,2, \ldots, N$ is the index of the flavor global $\mathrm{SU}(N)$ symmetry.

Prior to introduction of fermions the model has the form

$$
\mathcal{L}_{B}=-\frac{1}{4 g^{2}} \mathcal{F}_{\mu \nu}^{A} \mathcal{F}^{\mu \nu, A}+\mathcal{D}^{\mu} \bar{\phi}^{a} \mathcal{D}_{\mu} \phi_{a}-V(\phi)
$$

where $A=1, \ldots, N^{2}-1$ is the index of the adjoint representation for field strength tensors and summation over $A$ and $a$ is implied. The scalar sector has a global (flavor) $\mathrm{U}(N)$ symmetry with the potential $V(\phi)$ chosen exactly as in the appropriate supersymmetric field theories (see [14]),

$$
V=\lambda_{1} \sum_{A}\left|\sum_{a} \bar{\phi}^{a} T^{A} \phi_{a}\right|^{2}+\lambda_{2}\left|\sum_{a} \bar{\phi}^{a} \phi_{a}-N v^{2}\right|^{2} .
$$

Here $T^{A}$ are the generators of the color $\mathrm{SU}(N)$, and $\lambda_{1,2}$ are constants. This potential can be rewritten as

$$
V=\frac{\lambda_{1}}{2} \sum_{a, b}\left[\bar{\phi}^{a} \phi_{b} \bar{\phi}^{b} \phi_{a}-\frac{1}{N} \bar{\phi}^{a} \phi_{a} \bar{\phi}^{b} \phi_{b}\right]+\lambda_{2}\left|\sum_{a} \bar{\phi}^{a} \phi_{a}-N v^{2}\right|^{2} .
$$

It is minimized by the configuration (up to gauge transformation)

$$
\left\langle\phi_{a}^{i}\right\rangle=\mathrm{e}^{i \gamma} v \delta_{a}^{i}
$$

where the phase $\gamma$ is arbitrary. 
While all $N^{2}-1$ gauge bosons of the original $\mathrm{SU}(N)$ gauge theory are Higgsed [14, acquiring one and the same mass $M=g v$, one of Higgs fields remains massless representing a Goldstone boson associated with the global U(1) symmetry, $\phi \rightarrow$ $\exp (i \alpha) \phi$. The arbitrariness of $\gamma$ is just a reflection of this feature. To make our model similar to SM we would like to eliminate the single remaining massless mode. This can be done in three different ways.

First, we can add to the potential $V$ in Eq. (11) a gauge invariant term

$$
-\lambda_{3} \phi_{a_{1}}^{i_{1}} \phi_{a_{2}}^{i_{2}} \ldots \phi_{a_{N}}^{i_{N}} \varepsilon^{a_{1} a_{2} \ldots a_{N}} \varepsilon_{i_{1} i_{2} \ldots i_{N}}+\text { H.c. . }
$$

This term, a determinant of the matrix $\phi$, is also invariant under the global flavor $\mathrm{SU}(N)$ but it breaks the global $\mathrm{U}(1)$. It has dimension $N$ and is nonrenormalizable at $N>4$.

Second, we could expand the gauge sector adding a U(1) gauge boson (so that the gauge symmetry becomes $\mathrm{U}(N)$ rather than $\mathrm{SU}(N)$. Then it will be Higgsed too, and the overall $\phi$ field phase will be eaten. Needless to say, adding the $U(1)$ gauge will preclude us from introduction of the term (14).

Third, we could reduce the number of the fundamental Higgs fields from $N$ down to $N-1$.

Since the essence of our construction does not change, we will demonstrate it under the assumption that the term (14) is added to the potential, making $\gamma=0$ in Eq. (13).

Equation (13) shows that in the Higgs regime both the local gauge and global flavor groups are spontaneously broken, but the diagonal $\mathrm{SU}(N)$ survives as the exact global symmetry of the model.

Now we will construct the fermion sector. The simplest vectorlike example is given by one Dirac spinor $\Psi^{i}$ in the fundamental representation of $\mathrm{SU}(N)_{\text {gauge, }}$, which is equivalent to two left-handed Weyl spinors,

$$
\Psi^{i} \longrightarrow \chi_{\alpha}^{i}, \quad \tilde{\chi}_{i}^{\alpha}, \quad \alpha=1,2, \quad i=1, \ldots, N
$$

fundamental and anti-fundamental with respect to $\mathrm{SU}(N)_{\text {gauge }}$ and singlets with respect to the global $\mathrm{SU}(N)$. Besides there are two gauge-singlet Weyl fields which are anti-fundamental and fundamental with respect to the global $\mathrm{SU}(N)$,

$$
\eta_{a}^{\alpha}, \tilde{\eta}_{\alpha}^{a}, \quad \alpha=1,2, \quad a=1, \ldots, N
$$

Next, we will introduce the Yukawa terms

$$
\mathcal{L}_{\mathrm{Y}}=h_{1} \chi^{i} \bar{\phi}_{i}^{a} \eta_{a}+h_{2} \tilde{\chi}_{i} \phi_{a}^{i} \tilde{\eta}^{a}+\text { H.c. } .
$$


With the vacuum expectations value $\left\langle\phi_{a}^{i}\right\rangle=v \delta_{a}^{i}$ all fermions become massive; we have $2 N$ Dirac massive fermions. If $h_{1}=h_{2}=h$ their masses $h v$ are equal.

The Yukawa terms are invariant under the following phase rotations:

$$
\chi_{\alpha}^{i} \rightarrow \mathrm{e}^{i \alpha} \chi_{\alpha}^{i}, \quad \tilde{\chi}_{i}^{\alpha} \rightarrow \mathrm{e}^{i \tilde{\alpha}} \tilde{\chi}_{i}^{\alpha}, \quad \eta_{a}^{\alpha} \rightarrow \mathrm{e}^{-i \alpha} \eta_{a}^{\alpha}, \quad \tilde{\eta}_{\alpha}^{a} \rightarrow \mathrm{e}^{-i \tilde{\alpha}} \tilde{\eta}_{\alpha}^{a}
$$

where the gauge singlets and non-singlets are rotated in the opposite directions. The Higgs field $\phi$ stays intact. Then, the anomaly in the rotations of non-singlet fermions leads to the shift of the vacuum angle,

$$
\theta \longrightarrow \theta-N(\alpha+\tilde{\alpha})
$$

This makes the vacuum angle $\theta$ unobservable.

Note a crucial role of the extra U(1) given in Eq. (18) for this conclusion. If an extra mass term of the form $m \chi_{\alpha}^{i} \tilde{\chi}_{i}^{\alpha}$ is added the $\theta$ dependence reappears.

\subsection{Quiver generalizations}

It is not difficult to construct more sophisticated generalizations along these lines. Indeed, let us consider a standard "square" $\mathrm{SU}(N) \times \mathrm{SU}(N) \times \mathrm{SU}(N) \times \mathrm{SU}(N)$ quiver model depicted in Fig.1. This theory per se is chiral; hence no mass terms are possible. Generally speaking, we do not impose an extra $Z_{4}$ symmetry, so that the gauge couplings and vacuum angles may be different in the different $\mathrm{SU}(N)$ groups.

Of course, in the original version of the quiver theory all fermion fields are massless, and $\theta$ angles are unobservable. We can make them massive through Higgsing similar to that discussed in Sec.3.2. At each node we add a scalar field with one $\mathrm{SU}(N)_{\text {gauge }}$ index and one global, similar to (10). For instance, in the node 1 we add $\phi_{a_{1}}^{i_{1}}$ with the potential (11) 1 In addition, at the $1 \rightarrow 2$ link we add the spinor $\eta_{a_{1}}^{i_{2}}$ with the $\mathrm{SU}(N)_{\text {global }}$ index of the given node (i.e. 1 in the case at hand) and the $\mathrm{SU}(N)_{\text {gauge }}$ index of the next node (if we move clockwise). Then the gauge invariant Yukawa interaction for YM-1 has the form

$$
h_{1} \chi_{i_{2}}^{i_{1}} \bar{\phi}_{i_{1}}^{a_{1}} \eta_{a_{1}}^{i_{2}}
$$

Similar fields and interaction terms are added for YM-2, YM-3, and YM-4. Once all $\phi$ fields at each node develop expectation values (13), all four $\mathrm{SU}(N)$ gauge symmetries are spontaneously broken (i.e. the corresponding gauge bosons Higgsed). This

\footnotetext{
${ }^{1}$ The overall phase of the $\phi$ fields should be treated in the same way as in the previous section.
} 


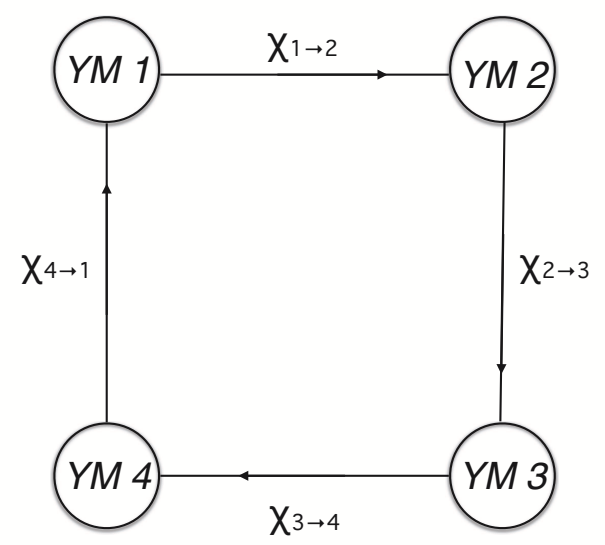

Figure 1: A "square" quiver $\mathrm{SU}(N) \times \mathrm{SU}(N) \times \mathrm{SU}(N) \times \mathrm{SU}(N)$. Each node represents a $\mathrm{SU}(N)$ Yang-Mills theory, and each link is a bifundamental fermion, with the first index in the fundamental representation of the first $\mathrm{SU}(N)$ while the second index in the antirepresentation of the second $\mathrm{SU}(N)$. For instance, on the upper horizontal link we have $\left(\chi_{1 \rightarrow 2}\right)_{i_{2}}^{i_{1}}$ and so on.

leaves us with only $\mathrm{SU}(N)_{\text {global }}$ at each node. All fermion fields acquire mass terms of the type

$$
\left(h_{1} v_{1}\right) \chi_{i_{2}}^{a_{1}} \eta_{a_{1}}^{i_{2}}
$$

Despite the absence of massless quarks or axions, the $\theta$-angles are unobservable. Indeed, we can rotate $\chi_{i_{2}}^{i_{1}}$ by a phase $\alpha_{1}$ while $\eta_{a_{1}}^{i_{2}}$ by $-\alpha_{1}$. The mass term (21) obviously stays intact. On the other hand, the vacuum angle $\theta_{1}$ of YM-1 is shifted, $\theta_{1} \rightarrow \theta_{1}-N \alpha_{1}$. Analogously, rotations of other $\chi$ and $\eta$ fields shift the angles $\theta_{p}$ of YM-p.

\section{Grand unification and $\theta$-dependence}

\subsection{SU(5) unification}

Let us consider the simplest SU(5) unification. In this theory a quark/lepton generation resides in one $\mathrm{SU}(5)$ decuplet $X_{\alpha}^{[a b]}$ and one antiquintet $\bar{V}_{\alpha, a}$ where $a=1,2, \ldots, 5$ is the $\mathrm{SU}(5)$ index. One weak lepton doublet $L_{\alpha}$ and three weak singlets $d_{\alpha, i}^{c}$ are form $\bar{V}_{\alpha}$ while other fields from (6), (17) enter $X$. 
At a large UV scale (let us call it $\left.v_{\mathrm{G}}\right) \mathrm{SU}(5)$ is broken down to $\mathrm{SU}(3) \times \mathrm{SU}(2) \times \mathrm{U}(1)$ by an adjoint Higgs field. This breaking produces 12 superheavy gauge bosons with masses $\sim g v_{\mathrm{G}}$. Then another Higgs field $\varphi^{a}$ (in the fundamental representation of $\mathrm{SU}(5))$ breaks $\mathrm{SU}(3) \times \mathrm{SU}(2) \times \mathrm{U}(1) \rightarrow \mathrm{SU}(3) \times \mathrm{U}(1)$ at the electroweak scale $v_{\mathrm{EW}}$. Three gauge bosons acquire masses $\sim g v_{\text {EW }}$.

Responsible for the masses of the matter fields Higgs is in the fundamental representation, while the adjoint Higgs plays no role in this process. We have the following Yukawa terms:

$$
\lambda_{1} \epsilon^{\alpha \beta} X_{\alpha}^{[a b]} \bar{V}_{\beta, a} \bar{\varphi}_{b}, \quad \lambda_{2} \epsilon^{\alpha \beta} X_{\alpha}^{[a b]} X_{\beta}^{[c d]} \varphi^{f} \varepsilon_{a b c d f} .
$$

At the scale above $v_{G}$ we have a unique $\theta$-term, see (11). The constants $\lambda_{1}$ and $\lambda_{2}$ may have phases too. The effective vacuum angle $\bar{\theta}$, independent of redefinition of phases, is

$$
\bar{\theta}=\theta+\arg \left(\lambda_{1} \lambda_{2}\right) .
$$

Indeed, in the second term one can absorb the phase of $\lambda_{2}$ in $\varphi$. Then, the phase of $\lambda_{1}$ changes, of course, $\arg \lambda_{1} \rightarrow \arg \lambda_{1}+\arg \lambda_{2}$. The latter phase can be eliminated by a redefinition of $\bar{V}$. Simultaneously, the $\theta$-angle changes as in Eq. (23). Thus, we can take $\lambda_{1,2}$ real, while $\bar{\theta}$ plays the role of our unique $\theta$ at the scale above $v_{G}$.

Now, let us consider the following phase rotations of the fields,

$$
X \rightarrow X e^{i \alpha_{X}}, \quad \bar{V} \rightarrow \bar{V} e^{i \alpha_{V}}, \quad \varphi \rightarrow \varphi e^{i \alpha_{\varphi}} .
$$

Due to chiral anomalies these rotations shift the $\theta$ angle by

$$
\theta \longrightarrow \theta-3 \alpha_{X}-\alpha_{V}
$$

The coefficient 3 for the decuplet immediately follows from its content of three SU(2) doublets versus one in the quintet. On the other hand, a simple examination of the $\mathrm{SU}(5)$ Yukawa terms (22) shows that the only classically conserved current in the theory at hand corresponds to

$$
\alpha_{V}=-3 \alpha_{X}, \quad \alpha_{\varphi}=-2 \alpha_{X}
$$

As it is seen from Eq. (25) this rotation is anomaly free and does not shift $\theta$, nor has it any impact on the Yukawa terms (22).

Thus, the parameter $\bar{\theta}$ is in principle observable in the $\mathrm{SU}(5)$ theory 2 What makes the situation different compared to the Standard Model? If we focus on the weak $\mathrm{SU}(2)$ corner of $\mathrm{SU}(5)$ we immediately see the difference with the Standard

\footnotetext{
${ }^{2} \mathrm{~A}$ similar conclusion was reached by V. Rubakov, private communication, 2016.
} 
Model: in $\mathrm{SU}(5)$ there exists a perturbative amplitude with $\Delta B=\Delta L= \pm 1$ generated by Higgs particle exchanges. The latter interferes with the instanton-induced amplitude.

Indeed, while the antiinstanton generates the vertex

$$
\left(X^{[a b]} X^{[c d]} X^{[f g]} V_{g} \varepsilon_{a b c d f}\right) e^{-i \theta} \exp \left(-\frac{8 \pi^{2}}{g^{2}}\right)
$$

(where a proper convolution of spinor indices is implied), an amplitude with the same combination of fields arises due to the $\varphi$ exchange through iterations of the mass terms (22). It has no $\theta$ and is proportional to $\lambda_{1} \lambda_{2}$. The interference of instantonic and perturbative pieces contains a proper combination

$$
\operatorname{Re}\left(\lambda_{1} \lambda_{2} \mathrm{e}^{i \theta}\right)=\left|\lambda_{1}\right|\left|\lambda_{2}\right| \mathrm{e}^{i \bar{\theta}}
$$

How the $\theta$ dependence shows up at low energies? An appropriate framework to approach the given problem is provided by the Wilson formalism. We start from a UV scale $\mu$ which is somewhat larger than $v_{G}$, and then evolve our "sliding scale" $\mu$ down. In doing so we calculate the coefficients in front of various operators which appear in the Wilson Lagrangian as a function of $\mu$. As long as $\mu \gg v_{G}$ we can ignore symmetry breaking. Below $v_{G}$ we will have to take into account the two-step symmetry breaking, at $v_{G}$ and at $v$.

At $\mu \gg v_{G}$ instantons with the sizes $\rho<1 / \mu$ generate the operator (27) with the running $g^{2}(\mu)$ and an extra coefficient $1 / \mu^{2}$ (leaving aside a certain power of the coupling constant $g^{2}$ ). This is the 't Hooft interaction generated by SU(5) instantons which is unambiguously determined. It was observed long ago that small-size instantons, i.e. $\rho \ll 1 / v_{G}$, probe the entire gauge group $\mathrm{SU}(5)$ irrespective of the spontaneous breaking which may or may not occur at low energies [10] (for a recent discussion see [11]).

Once $\mu$ hits $v_{G}$ the subsequent downward evolution splits in two flows: in the $\mathrm{SU}(3)$ and $\mathrm{SU}(2)$ corners, respectively. The operator (27) also splits in two terms, Eq. (9) in the $\mathrm{SU}(2)$ corner (with $\theta_{\mathrm{EW}}=\bar{\theta}$ ) and

$$
u d u^{c} d^{c} e^{i \bar{\theta}} \exp \left(-\frac{8 \pi^{2}}{g^{2}}\right) \text { in the } \mathrm{SU}(3) \text { corner, }
$$

with the color and spinor indices appropriately convoluted to form a singlet operator. The role and fate of (29) is well-known. In particular, its interference with mass terms leads to the $\theta$ dependence and $\mathrm{CP}$ violation at low scales. 
It is worth emphasizing that we have one and the same $\theta$ for the $\mathrm{SU}(3)$ and $\mathrm{SU}(2)$ group factors, in contradistinction to the bottom up approach of Ref. [15] 3$]$

This conclusion is valid even when other sources of $C P$ violation are present leading to a running of $\bar{\theta}$. This happens, in particular, when we deal with more than one generation. Couplings $\lambda_{1,2}$ become matrices with the generation indices, generally speaking containing $C P$ breaking phases. Higher order iterations in these $\lambda_{1,2}$ matrices produce a logarithmic running of $\bar{\theta}$ similar to that exhibited in the calculation [16] in SM. For several generations the definition (23) of $\bar{\theta}$ must be modified in an obvious way: $\lambda_{1,2}$ must be replaced by determinants of these matrices.

Above we explained that the $\theta$ dependence in the $\mathrm{SU}(2)$ corner shows up the in $\Delta B=\Delta L= \pm 1$ processes due do interference with perturbative superheavy Higgs exchanges. It implies a suppression $m_{W}^{2} / m_{\Phi}^{2}$ for the interference besides the exponential smallness, $\exp \left(-8 \pi^{2} / g^{2}\left(m_{W}\right)\right)$. As was mentioned above, thermal enhancement of the instanton contribution in early Universe noted in [13] may compensate, perhaps partly, the above suppression.

\subsection{Axion}

Now let us say a few words about the axion mechanism. The axion mechanism could be set up at the extreme UV scale, say, by strings. Then it is natural to expect that the axion coupling at $v_{G}$ is

$$
-\frac{a}{32 \pi^{2} f} \mathcal{F}_{\mu \nu}^{A} \tilde{\mathcal{F}}^{\mu \nu, A}
$$

where $\mathcal{F}_{\mu \nu}^{A}$ is the gauge field strength tensor for $\mathrm{SU}(5)$ and $A$ is the adjoint $\mathrm{SU}(5)$ index. If so, in Eq. (27)

$$
\theta \rightarrow \theta-a / f
$$

and the nonperturbative axion mass generated in the $\mathrm{SU}(3)$ corner makes the effective $\theta$ to vanish in both $\mathrm{SU}(3)$ and $\mathrm{SU}(2)$ corners simultaneously.

\section{Conclusions}

The $\theta$ angle implications in Yang-Mills theories at low energies crucially depend on the structure of the theory in the ultraviolet. Depending on details, $\theta$ independence can emerge without massless quarks and/or axions. In this paper we prove that the

\footnotetext{
${ }^{3}$ Note, however, that in the above publication UV completion was not considered.
} 
Anselm-Johansen example can be generalized and, in fact, covers a broad class of Yang-Mills theories in the Higgs regime.

Further consequences follow from unification at a high scale. For instance, in the SU(5) Grand Unification $\theta$-related effects in the SU(2) corner of the GlashowWeinberg-Salam model become observable, despite the fact that in the GlashowWeinberg-Salam model per se they are not. The very fact of unification implies that after possible spontaneous breaking $G \rightarrow G_{1} \times G_{2} \times \ldots$ all $G_{1,2, \ldots}$ subgroups inherit one and the same $\bar{\theta}$.

In general, if at a high scale in the ultraviolet, where "our" physics is set up, there is a unification (with a single unifying gauge group), and the ultraviolet analysis using no data on the subsequent evolution to low energies shows that there is no $\theta$ dependence, this statement will remain valid at low energies although the implementation mechanisms may vary. Two most popular mechanisms - massless quarks and axions - should be supplemented in the Higgs regime by generalizations of the Anselm-Johansen mechanism. Relatively close scenarios are those based on the spontaneous breaking of $C P$ invariance.

\section{Acknowledgments}

We are grateful to Z. Berezhiani, G. Dvali and V. Rubakov for discussions pertinent to Sect.4.

The work of M.S. was supported in part by DOE grant DE-FG02-94ER40823. A.V. appreciates hospitality of the Kavli Institute for Theoretical Physics where his research was supported in part by the National Science Foundation under Grant No. NSF PHY-1125915. 


\section{References}

[1] R. Jackiw and C. Rebbi, Phys. Rev. Lett. 37, 172 (1976) [reprinted in M. Shifman (Ed.), Instantons in Gauge Theories, (World Scientific, Singapore, 1994), p. 25].

[2] C. G. Callan, R. F. Dashen and D. J. Gross, Phys. Lett. B 63, 334 (1976) [reprinted in M. Shifman (Ed.), Instantons in Gauge Theories, (World Scientific, Singapore, 1994), p. 29].

[3] M. A. Shifman, A. I. Vainshtein and V. I. Zakharov, Can Confinement Ensure Natural CP Invariance of Strong Interactions?, Nucl. Phys. B 166, 493 (1980).

[4] R. D. Peccei and H. R. Quinn, CP Conservation in the Presence of Instantons, Phys. Rev. Lett. 38, 1440 (1977).

[5] S. Weinberg, A New Light Boson?, Phys. Rev. Lett. 40, 223 (1978);

F. Wilczek, Problem of Strong P and T Invariance in the Presence of Instantons, Phys. Rev. Lett. 40, 279 (1978).

[6] A. E. Nelson, Naturally Weak CP Violation, Phys. Lett. 136B, 387 (1984); Calculation of $\theta$ Barr, Phys. Lett. 143B, 165 (1984).

[7] S. M. Barr, Solving the Strong CP Problem Without the Peccei-Quinn Symmetry, Phys. Rev. Lett. 53, 329 (1984); A Natural Class of Non-Peccei-Quinn Models, Phys. Rev. D 30, 1805 (1984).

[8] A. A. Anselm and A. A. Johansen, Baryon nonconservation in standard model and Yukawa interaction, Nucl. Phys. B 407, 313 (1993); Can electroweak $\theta$ term be observable?, Nucl. Phys. B 412, 553 (1994), hep-ph/9305271.

[9] P. Fileviez Perez and H. H. Patel, The Electroweak Vacuum Angle, Phys. Lett. B 732, 241 (2014) [arXiv:1402.6340 [hep-ph]].

[10] S. Dimopoulos, A Solution of the Strong CP Problem in Models With Scalars, Phys. Lett. B 84, 435 (1979).

[11] T. Gherghetta, N. Nagata and M. Shifman, A Visible QCD Axion from an Enlarged Color Group, Phys. Rev. D 93, 115010 (2016), [arXiv:1604.01127] [hep-ph]].

[12] G. 't Hooft, Computation of the Quantum Effects Due to a Four-Dimensional Pseudoparticle, Phys. Rev. D 14, 3432 (1976) Erratum: [Phys. Rev. D 18, 2199 (1978)]. 
[13] A. Polyakov, Models and Mechanisms in Gauge Theory, in Proc. 9the Int. Symp. on Lepton and Photon Interactions at High Energy, Batavia, IL, 1979, Eds. T.B.W. Kirk et al., p. 521 [https://inspirehep.net/record/149270/files/C790823-pg.520.PDF ];

V. A. Kuzmin, V. A. Rubakov and M. E. Shaposhnikov, On the Anomalous Electroweak Baryon Number Nonconservation in the Early Universe, Phys. Lett. 155B, 36 (1985).

P. B. Arnold and L. D. McLerran, Sphalerons, Small Fluctuations and Baryon Number Violation in Electroweak Theory, Phys. Rev. D 36, 581 (1987); The Sphaleron Strikes Back, Phys. Rev. D 37, 1020 (1988).

[14] R. Auzzi, S. Bolognesi, J. Evslin, K. Konishi and A. Yung, Non-Abelian superconductors: Vortices and confinement in $\mathcal{N}=2 S Q C D$, Nucl. Phys. B 673, 187 (2003) hep-th/0307287;

M. Shifman and A. Yung, Non-Abelian string junctions as confined monopoles, Phys. Rev. D 70, 045004 (2004) hep-th/0403149.

M. Shifman and A. Yung, Supersymmetric Solitons, (Cambridge University Press, 2009).

[15] G. Dvali, Three-form Gauging of Axion Symmetries and Gravity, hep-th/0507215, and private communications (2016).

[16] J. R. Ellis and M. K. Gaillard, Strong and Weak CP Violation, Nucl. Phys. B 150, 141 (1979). 